\title{
Long-term outcome of CBT in adults with OCD and comorbid ASD: A naturalistic follow-up study
}

\author{
Akiko Nakagawa $^{1} \cdot$ Nora Choque Olsson $^{2}$ (D) Yoko Hiraoka $^{3} \cdot$ Hirofumi Nishinaka ${ }^{4}$. Tetsuji Miyazaki ${ }^{3} \cdot$ Naoko Kato $^{1}$. \\ Eriko Nakatani $^{1,5} \cdot$ Mayumi Tomita $^{6} \cdot$ Kazuko Yoshioka $^{7} \cdot$ Shinji Murakami $^{3} \cdot$ Shozo Aoki $^{3}$
}

Published online: 19 September 2018

(C) The Author(s) 2018

\begin{abstract}
Obsessive-compulsive disorder (OCD) and autism spectrum disorders (ASD) are highly comorbid. In adults with OCD, cognitive-behavioral therapy (CBT) has been extensively demonstrated to be an effective treatment. However, to date, no follow-up studies exist regarding the long-term outcome of CBT in adults with OCD with comorbid ASD in a regular and naturalistic clinical setting. In the current study, we aimed to study the long-term outcome of CBT among OCD patients with and without ASD. In total, 30 patients diagnosed with OCD who received CBT at a naturalistic psychiatric clinic in Japan were included in the follow-up study. The patients were divided into two groups: OCD with ASD and OCD without ASD. The results revealed no group differences regarding age, gender and OCD symptom severity at baseline. The Yale-Brown ObsessiveCompulsive Scale total score (primary outcome) at follow-up showed decreased severity of symptoms in both groups, thus indicating both groups responded to CBT. However, no significant differences were evident between the groups. Overall, the results suggest that even adults with OCD with comorbid ASD benefit from CBT. A survey on patients' impressions of treatment effect at follow-up showed high rates of satisfaction with CBT and most participants attributed their improvement to CBT as opposed to pharmacological treatment. This study showed the outcome differences of OCD symptom dimensions in OCD with ASD patients compared with the OCD without ASD patients. These differences suggest the need for developing adapted CBT for adults with OCD with ASD and for optimizing the treatment and their lives.
\end{abstract}

Keywords Obsessive-compulsive disorder · Autism spectrum disorder · Cognitive-behavioral therapy · Follow-up study · Naturalistic setting

Electronic supplementary material The online version of this article (https://doi.org/10.1007/s12144-018-9952-1) contains supplementary material, which is available to authorized users.

Nora Choque Olsson

nora.choque-olsson@ki.se

1 Research Center for Child Mental Development, Chiba University, Chiba, Japan

2 Centre for Psychiatry Research, Department of Clinical Neuroscience, Karolinska Institutet, Stockholm, Sweden

3 Department of Psychiatry, Kawasaki Medical School, Kurashiki, Japan

4 Center for Forensic Mental Health, Chiba University, Chiba, Japan

5 Department of Psychiatry, Faculty of Medicine, Kyshu University, Fukuoka, Japan

6 Faculty of Literature, Kurume University, Kurume, Japan

7 School of Human and Social Science, Fukuoka Prefectural University, Tagawa, Japan

\section{Introduction}

Obsessive-compulsive disorder (OCD) is characterized by two features. Obsessions are defined as recurrent, unwanted thoughts, urges, or images that are experienced as intrusive. Compulsions are repetitive behaviors or mental acts executed to reduce and/or neutralize anxiety related with obsessions (American Psychiatric Association 2013). OCD is one of the World Health Organization's top 20 leading causes of considerable suffering and disease burden among individuals aged 15-44 years (Kohn et al. 2004). In addition, OCD has a substantial negative impact on daily life, affecting occupational or academic functioning and social and familial relationships (Piacentini et al. 2003; Mito et al. 2014).

Autism spectrum disorder (ASD) is a lifelong neurodevelopmental disorder. It is characterized by deficits in social communication and interaction, repetitive and stereotyped behavior, and restricted interests (American Psychiatric 
Association 2013). ASD can co-occur with OCD in both children and adults (Griffiths et al. 2017). A recent study showed that individuals with a primary diagnosis of OCD had almost a fourfold higher risk of an ASD diagnosis later in life, and individuals with a primary diagnosis of ASD had a twofold higher risk of comorbid OCD (Meier et al. 2015). Other studies have shown high rates of ASD among individuals with OCD (Arildskov et al. 2016; Bejerot 2007; Bejerot et al. 2001; Ruta et al. 2010), indicating the importance of assessing ASD symptoms in treatment-resistant individuals with OCD (Bejerot 2007; Mito et al. 2014).

Cognitive-behavioral therapy (CBT) is a promising treatment for individuals with OCD (O'Neill and Feusner 2015; Sharma et al. 2014). Although several studies have focused on the treatment outcome of CBT in individuals with OCD, few studies have investigated long-term treatment outcomes in OCD patients with ASD (Murray et al. 2015; Russell et al. 2013). Some study findings suggest that OCD patients with ASD show less improvement after CBT treatment than those without ASD (Murray et al. 2015; Sharma et al. 2014); however, other studies indicate that CBT has good treatment efficacy for individuals with OCD and ASD (Russell et al. 2013) and for those with anxiety symptoms (White et al. 2013; Maddox et al. 2016; Walters et al. 2016). A recent review on effectiveness of CBT for individuals with ASD and comorbid OCD indicated that a standard CBT protocol enhanced with modifications such as parental involvement, increased use of visual stimuli, personalized treatment metaphors, self-monitoring, positive reinforcement, and use of clear language and instructions was most effective (Kose et al. 2018). However, there are few studies on the long-term outcome of CBT for OCD patients with ASD in a naturalistic clinical setting.

Most OCD patients in Japan have difficulty accessing CBT because of the serious shortage of CBT therapists, particularly in local areas. OCD patients in Japan are referred to specialized centers for CBT if they fail to improve sufficiently after pharmacotherapy treatment and/or other psychotherapies. During CBT treatment, comorbid ASD is often detected in individuals who are drug resistant. As ASD is a lifelong condition, it is clinically important to investigate whether the effect of CBT is maintained in drug resistant OCD patients with comorbid ASD. Therefore, the first author (AN) conducted a study to investigate the clinical characteristics of adult OCD patients with comorbid ASD and those without comorbid ASD. The study was conducted at the Department of Psychiatry, Kawasaki Medical School Hospital, Kurashiki, Japan, between 2008 and 2011. After reporting the preliminary results of the study (Yamashita 2010), the research group continued the research, and provided naturalistic CBTtreatment to patients with OCD, until 2011.

The objective of this follow-up study was to investigate the long-term outcome of CBT treatment in OCD patients with ASD in a naturalistic psychiatric setting in Japan, and to investigate their impressions of the treatment effect.

\section{Methods}

\section{Participants}

All 64 patients who participated in the previous study described above were invited to participate in this follow-up study, from which 30 patients entered. Some participants had OCD with comorbid ASD and others had OCD without comorbid ASD; the two groups are referred to here as OCD (ASD+) and OCD (ASD-), respectively.

The eligibility criteria for the first assessment were patients aged 18-60 years with a clinical diagnosis of OCD according to the Structured Clinical Interview for the Diagnostic and Statistical Manual of Mental Disorders, version IV (SCIDIV) (Otsubo et al. 2005) for OCD and a total score of $\geq 16$ on the Yale-Brown Obsessive Compulsive Scale, Y-BOCS (Goodman et al. 1989), and an IQ score of $\geq 80$ on the Wechsler Intelligence Scale (Wechsler 1997). The ASD diagnosis was confirmed by two experienced psychiatrists using the DSM-IV (American Psychiatric Association 2000), the parent-rated Pervasive Developmental Disorders Autism Society Japan Rating Scale, PARS (Kamio et al. 2006), and the Autism Spectrum Quotient Japanese version, AQ-J (Baron-Cohen et al. 2001; Wakabayashi et al. 2006). Depressive symptoms were assessed using the Beck Depression Inventory, Second Edition, and BDI-II (Beck and Steer 1996; Kojima et al. 2002). Participants were excluded if they had current severe depression, schizophrenia, a head injury, serious medical conditions, or alcohol addiction.

The assessment at follow-up was conducted 4-11 years after the initial assessment. All patients who had participated in the previous study and had continued to receive CBT were asked to participate in the follow-up study. The patients received an invitation letter with information about the study, consent forms, outcome questionnaires (self-report Y-BOCS symptom severity scale and the BDI-II), and a survey questionnaire about current status and impression of treatment effect (Appendix). A total of 30 patients completed the questionnaires and the survey. The institutional research and ethics committee of Kawasaki Medical School Hospital, Japan, approved all study protocols, including the follow-up survey (No. 1452). Written informed consent was obtained from all study participants.

\section{Treatment}

All participants were CBT-naïve and they continued to take any medication previously prescribed by psychiatrists. The CBT treatment was individually tailored and included an agenda, psychoeducation, accurate behavioral analysis, exposure and response prevention (ERP) if applicable, homework, and relapse prevention. All participants received ERP after accurate behavioral analysis except 6 patients who were found to have 
no OC symptoms for which ERP was applicable. All these 6 patients were in the ASD (OCD+) group.

The therapist group comprised seven psychiatrists with over 4 years of clinical experience and 1-4 years of CBT experience, except for the first author (AN). AN is a senior psychiatrist with more than 30 years of experience using CBT for OCD. AN supervised the junior therapists and provided therapy. The junior therapists participated in individual and group supervision led by AN to facilitate the discussion of therapeutic difficulties, provide skills acquisition, and encourage peer support and interaction. In the group supervision, accurate behavioral analysis (functional behavioral assessment) was considered the most important CBT component for OCD. Behavioral analysis was performed for each patient to ensure that the appropriate treatment procedures were applied. Attention was paid not only to patients' obsessive and compulsive (OC) symptoms, but also to ASD symptoms such as difficulties in verbal and non-verbal communication, hyperor hyposensitivity, and difficulties adapting to a new environment. The standard CBT for OCD was modified for OCD (ASD+) such as increased involvement of caregivers, use of visual stimuli in psychoeducation, self-monitoring, positive reinforcement, and use of clear language and instructions. Although the treatment was not controlled systematically, we believe that it showed good adherence because the therapists were supervised by an experienced CBT therapist.

The CBT active period for the participants was between 2008, the comparison study started, and 2011. After AN left the department early in 2012, 33 patients of the 64 were referred to local psychiatrists who did not practice CBT and 20 patients were followed up by trained junior psychiatrists in the same hospital. The other 11 patients terminated their treatment before AN left.

\section{Outcome Measures}

\section{Primary Outcome}

The Yale-Brown Obsessive Compulsive Scale (Y-BOCS) (Goodman et al. 1989) was used to measure severity of OCD symptoms. This measure is not influenced by the type of obsessions or compulsions present. The scale comprises 10 items (five obsession and five compulsion items). Each item is rated from 0 (no symptoms) to 4 (extreme symptoms); the total range is $0-40$ and there are separate subtotals for severity of obsessions and compulsions. The Y-BOCS showed excellent interrater reliability (intraclass correlation coefficients above $r=0.85$ ) and a high degree of internal consistency among all item scores, indicated by Cronbach's alpha coefficient (Goodman et al. 1989). The scale was used in a semistructured interview setting.

The Yale-Brown Symptom Checklist was also used to identify the content of each patient's obsessions (subscales:
Aggressive, Contamination, Sexual, Hoarding, Religious, Symmetry, Miscellaneous, Somatic) and compulsions (subscales: Cleaning, Checking, Repeating, Counting, Arranging, Hoarding, Miscellaneous).

\section{Secondary Outcomes}

Beck Depression Inventory-Second Edition (BDI-II) (Beck and Steer 1996). Subjective severity of depression was assessed using the BDI-II. The BDI-II has been translated into Japanese and the linguistic equivalence verified by back-translation. The Japanese version of the BDI-II shows a high level of internal consistency reliability (Cronbach's alpha $=0.87$ ) (Kojima et al. 2002).

\section{Survey of Patients' Impressions of Treatment Effect, Treatment Satisfaction, and Attribution at Follow-Up and Current Diagnosis}

Participants completed a questionnaire on treatment satisfaction and their experiences of the CBT treatment. The survey included questions about their social and economic conditions (e.g., who they lived with, their employment, partner or close friends, and current CBT treatment) (Appendix). The survey also included questions about how much patients valued the treatment effect on a scale of 1 to $7(1=$ very much worse; $2=$ much worse; 3 = a little worse; $4=$ unchanged; $5=$ a little better; $6=$ much better; $7=$ very much better) and questions related to participants' attribution of their improvement (CBT or pharmacological treatment). Information about each patient's current SCID-based OCD diagnosis at follow-up was obtained from their psychiatrists.

\section{Statistical Analysis}

Student's t-tests for continuous data and Fisher's exact tests for categorical data were used to examine group differences. Analysis of variance (ANOVA) was performed at pretreatment and follow-up to analyze treatment outcomes in both groups. To detect changes in symptoms, two-way ANOVA was conducted for "group" $\times$ "time" (i.e., OCD (ASD+), OCD $\left(\mathrm{ASD}^{-}\right) \times$pre-treatment and follow-up, respectively). To measure differences between repliers and non-repliers, we used Student's t-test for each group at the first assessment.

\section{Results}

\section{Participant Characteristics at the First Assessment}

Table 1 shows the sample characteristics at the first assessment. No significant differences between the groups were found for age, gender, IQ, or OCD symptoms. Nevertheless, 
Table 1 Sample characteristics at the first assessment: OCD (ASD -) versus (ASD+)

\begin{tabular}{lllll}
\hline & $\begin{array}{l}\text { OCD (ASD-) } N=45 \\
\text { Mean } \pm \text { SD }\end{array}$ & $\begin{array}{l}\text { OCD (ASD+) } N=19 \\
\text { Mean } \pm \text { SD }\end{array}$ & t & P \\
\hline Age & $34.09 \pm 9.41$ & $33.16 \pm 8.67$ & 0.370 & 0.713 \\
Sex (F/M) & $30 / 15$ & $10 / 9$ & - & $0.171^{\text {a }}$ \\
Age of onset & $22.22 \pm 9.68$ & $19.84 \pm 6.25$ & 0.986 & 0.328 \\
Y-BOCS & & & & \\
Total & $31.47 \pm 6.89$ & $33.79 \pm 5.46$ & 1.304 & 0.197 \\
Y-BOCS Obsessive & $15.49 \pm 3.72$ & $16.74 \pm 2.86$ & 1.305 & 0.197 \\
Y-BOCS Compulsive & $15.98 \pm 3.56$ & $17.16 \pm 2.71$ & 1.292 & 0.201 \\
PARS & $(N=35)$ & $(N=18)$ & & \\
Childhood (cut off: 9) & $3.31 \pm 3.24$ & $9.72 \pm 7.15$ & 3.615 & $\mathbf{0 . 0 0 2}$ \\
Adolescent and adult (cut off: 20$)$ & $10.11 \pm 6.94$ & $21.83 \pm 8.72$ & 5.329 & $<\mathbf{0 . 0 0 1}$ \\
AQ & & & & \\
AQ-J total (cut off: 33) & $23.76 \pm 7.15$ & $28.47 \pm 7.81$ & 2.347 & 0.022 \\
WAIS-III & & & & \\
FIQ & $100.67 \pm 13.85$ & $102.68 \pm 14.48$ & 0.525 & 0.601 \\
VIQ & $103.42 \pm 13.70$ & $108.32 \pm 13.61$ & 1.308 & 0.196 \\
PIQ & $97.09 \pm 13.56$ & $94.89 \pm 15.15$ & 0.571 & 0.570 \\
Depression & & & & \\
BDI-II & $15.27 \pm 8.37$ & $15.21 \pm 10.72$ & 0.023 & 0.982 \\
\hline
\end{tabular}

Bold numbers show statistical significance

ASD, Autism Spectrum Disorders; AQ, Autism Spectrum Quotient; AQ-J, Autism Spectrum Quotient Japanese version; BDI, Beck Depression Inventory; FIQ, Full Scale IQ; OCD, Obsessive Compulsive Disorder; OCD (ASD-), Obsessive compulsive disorder without co-morbid autism spectrum disorders; (ASD+), Obsessive compulsive disorder with co-morbid autism spectrum disorders; PIQ, Performance IQ; PARS, Pervasive Developmental Disorders Autism Society Japan Rating Scale; VIQ, Verbal IQ; WAIS-III, Wechsler Intelligence Scale- third edition; Y-BOCS, Yale-Brown Obsessive-Compulsive Scale

${ }^{a}$ Fisher's exact test

significant differences were observed in scores on the ASDrelated scales. The OCD (ASD+) had higher total AQ-J scores than the OCD (ASD-) group. Similar between-group differences were found for the PARS childhood and PARS adolescent and adult scores. Regarding depression symptoms, there were no between-group differences in BDI-II scores (Table 1).

Table 2 shows the OC symptom differences at the first assessment according to the Y-BOCS Symptom Checklist. The rates of hoarding symptoms on the Obsession subscale were lower in the OCD (ASD-) group than in the OCD (ASD+) group. Similar results were found for symmetry symptoms. There were also symptom differences on the Compulsion subscale: the OCD (ASD-) group showed lower rates of repeating, arranging, and hoarding symptoms than the OCD (ASD+) group (Table 2).

\section{Results at Follow-Up}

In total, 30 of the 64 patients, 18 OCD (ASD-) and 12 OCD (ASD+), responded to the follow-up assessment. Of the 34 non-repliers, 12 did not receive the follow-up questionnaire (7 patients had moved residence, 3 patients' letters with the follow-up questionnaires were returned to the researchers, and in the case of 2 patients, one of their parents declined participation in the follow-up study); 22 patients did not provide a reason for not responding.

Comparisons of the demographic profiles and clinical characteristics of the two groups at follow-up are shown in Tables 3 and 4. For both groups, the results at follow-up showed that most patients lived with someone and that there was no difference between the groups. As expected, the rates of having friends and partners were higher in the OCD (ASD -) group than in the OCD (ASD+) group, but the difference was not statistically significant. Interestingly, the rate of employment (including in sheltered workplaces) was higher in the OCD (ASD+) group than in the OCD (ASD-) group, but the results were not statistically significant.

Regarding current OCD diagnosis, more patients in the OCD (ASD+) group maintained an OCD diagnosis than did patients in the OCD (ASD-) group, but the difference was not significant. Further, more patients in the OCD (ASD+) group received psychiatric care compared with patients in the OCD (ASD-) group, although the difference was not significant (Table 3). In both groups, almost all patients who received psychiatric treatment were taking medication: 11 out of 12 in OCD (ASD+) and 12 out of 18 in OCD (ASD-). In the 
Table 2 Yale-Brown Obsessive-Compulsive Scale symptom checklist at the first assessment: $\mathrm{OCD}(\mathrm{ASD}-)$ versus (ASD+)

$\frac{\mathrm{OCD}(\mathrm{ASD}-)}{(-) \quad(+) \quad \%} \frac{\mathrm{OCD}(\mathrm{ASD}+)}{(-) \quad(+) \quad \%} P^{\mathrm{a}}$

\begin{tabular}{llllllll}
\hline Obsession & & & & & & & \\
Aggressive & 22 & 23 & $51.1 \%$ & 5 & 14 & $73.7 \%$ & 0.107 \\
Contamination & 13 & 32 & $71.1 \%$ & 7 & 12 & $63.2 \%$ & 0.565 \\
Sexual & 43 & 2 & $4.4 \%$ & 17 & 2 & $10.5 \%$ & 0.576 \\
Hoarding & 34 & 11 & $24.4 \%$ & 8 & 11 & $57.9 \%$ & $\mathbf{0 . 0 2 0}$ \\
Religious & 37 & 8 & $17.8 \%$ & 15 & 4 & $21.1 \%$ & 0.739 \\
Symmetry & 31 & 14 & $31.1 \%$ & 8 & 11 & $57.9 \%$ & 0.055 \\
Miscellaneous & 14 & 31 & $68.9 \%$ & 2 & 17 & $89.5 \%$ & 0.117 \\
Somatic & 42 & 3 & $6.7 \%$ & 15 & 4 & $21.1 \%$ & 0.182 \\
Compulsion & & & & & & & \\
Cleaning & 12 & 33 & $73.3 \%$ & 5 & 14 & $73.7 \%$ & 1.000 \\
Checking & 18 & 27 & $60.0 \%$ & 3 & 16 & $84.2 \%$ & 0.082 \\
Repeating & 25 & 20 & $44.4 \%$ & 4 & 15 & $78.9 \%$ & $\mathbf{0 . 0 1 4}$ \\
Counting & 42 & 3 & $6.7 \%$ & 16 & 3 & $15.8 \%$ & 0.351 \\
Arranging & 38 & 7 & $15.6 \%$ & 11 & 8 & $42.1 \%$ & 0.049 \\
Hoarding & 34 & 11 & $24.4 \%$ & 7 & 12 & $63.2 \%$ & $\mathbf{0 . 0 0 5}$ \\
Miscellaneous & 28 & 17 & $37.8 \%$ & 10 & 9 & $47.4 \%$ & 0.580 \\
\hline
\end{tabular}

Bold numbers show statistical significance

OCD (ASD-), Obsessive compulsive disorder without co-morbid autism spectrum disorders; (ASD+), Obsessive compulsive disorder with comorbid autism spectrum disorders

${ }^{a}$ Fisher's exact test
Table 4 Yale-brown obsessive-compulsive scale symptom checklist of repliers at the first assessment: OCD (ASD-) versus (ASD+)

$$
\frac{\mathrm{OCD}(\mathrm{ASD}-)}{(-) \quad(+) \quad \%} \frac{\mathrm{OCD}(\mathrm{ASD}+) N=12}{(-) \quad(+) \%} P^{\mathrm{a}}
$$

\begin{tabular}{llllllll}
\hline Obsession & & & & & & & \\
Aggressive & 10 & 8 & $44.4 \%$ & 2 & 10 & $83.3 \%$ & 0.058 \\
Contamination & 4 & 14 & $77.8 \%$ & 4 & 8 & $66.7 \%$ & 0.678 \\
Sexual & 17 & 1 & $5.6 \%$ & 11 & 1 & $8.3 \%$ & 1.000 \\
Hoarding & 14 & 4 & $22.2 \%$ & 4 & 8 & $66.7 \%$ & $\mathbf{0 . 0 2 4}$ \\
Religious & 13 & 5 & $27.8 \%$ & 10 & 2 & $16.7 \%$ & 0.669 \\
Symmetry & 13 & 5 & $27.8 \%$ & 3 & 9 & $75.0 \%$ & $\mathbf{0 . 0 2 4}$ \\
Miscellaneous & 3 & 15 & $83.3 \%$ & 1 & 11 & $91.7 \%$ & 0.632 \\
Somatic & 17 & 1 & $5.6 \%$ & 9 & 3 & $25.0 \%$ & 0.274 \\
Compulsion & & & & & & & \\
Cleaning & 4 & 14 & $77.8 \%$ & 4 & 8 & $66.7 \%$ & 0.678 \\
Checking & 9 & 9 & $50.0 \%$ & 2 & 10 & $83.3 \%$ & 0.121 \\
Repeating & 9 & 9 & $50.0 \%$ & 2 & 10 & $83.3 \%$ & 0.121 \\
Counting & 18 & 0 & $0.0 \%$ & 9 & 3 & $25.0 \%$ & 0.054 \\
Arranging & 17 & 1 & $5.6 \%$ & 5 & 7 & $58.3 \%$ & $\mathbf{0 . 0 0 3}$ \\
Hoarding & 14 & 4 & $22.2 \%$ & 4 & 8 & $66.7 \%$ & $\mathbf{0 . 0 2 4}$ \\
Miscellaneous & 9 & 9 & $50.0 \%$ & 4 & 8 & $66.7 \%$ & 0.465 \\
\hline
\end{tabular}

Bold numbers show statistical significance

OCD, Obsessive Compulsive Disorder; OCD (ASD-), Obsessive compulsive disorder without co-morbid autism spectrum disorders; (ASD+), Obsessive compulsive disorder with co-morbid autism spectrum disorders

${ }^{\text {a }}$ Fisher's exact test
Table 3 Demographic profiles and clinical characteristics at follow-up: OCD (ASD-) versus (ASD+)

\begin{tabular}{lllll}
\hline & OCD (ASD-) & OCD (ASD+) & t & P \\
& $N=18$ & & \\
& Mean \pm SD & Mean \pm SD & & \\
\hline Age & $44.78 \pm 11.10$ & $39.42 \pm 11.06$ & -1.298 & 0.205 \\
Sex (F/M) & $14 / 4$ & $5 / 7$ & - & $0.063^{\mathrm{a}}$ \\
Age of onset & $23.33 \pm 11.78$ & $22.17 \pm 5.91$ & 0.316 & 0.754 \\
Duration from first medical examination & $86.67 \pm 28.38$ & $84.50 \pm 22.68$ & 0.221 & 0.827 \\
$\quad$ (months) & $0 / 18$ & $1 / 11$ & - & $0.400^{\mathrm{a}}$ \\
Residence form (single/others) & $11 / 7$ & $4 / 8$ & - & $0.264^{\mathrm{a}}$ \\
Partner (Presence/absence) & $12 / 6$ & $4 / 8$ & - & $0.135^{\mathrm{a}}$ \\
Friendes (Presence/absence) & $9 / 9$ & $8 / 4$ & - & $0.465^{\mathrm{a}}$ \\
Job status (employment/unemployment) & $10 / 8$ & $5 / 7$ & - & $0.710^{\mathrm{a}}$ \\
Hobbies (Presence/absence) & $10 / 8$ & $9 / 3$ & - & $0.442^{\mathrm{a}}$ \\
Current diagnosis of OCD by SCID & $13 / 5$ & $11 / 1$ & - & $0.358^{\mathrm{a}}$ \\
$\quad$ Presence/absence) & $23.50 \pm 7.46$ & $28.08 \pm 8.64$ & -1.548 & $0.133^{\mathrm{a}}$ \\
Psychiatric care (Presence/absence) & & & - \\
AQ-J total (cut off: 33) & & & & - \\
\hline
\end{tabular}

ASD, Autism Spectrum Disorders; AQ, Autism Spectrum Quotient; AQ-J, Autism Spectrum Quotient Japanese version; OCD, Obsessive Compulsive Disorder; OCD (ASD-), Obsessive compulsive disorder without comorbid autism spectrum disorders; (ASD+), Obsessive compulsive disorder with co-morbid autism spectrum disorders

${ }^{a}$ Fisher's exact test 
OCD (ASD-) group, all patients took selective serotonin reuptake inhibitors (SSRIs). Four patients took only SSRIs and eight patients took a small dose of major tranquilizers as augmentation. None of the patients took only major tranquilizers. In the OCD (ASD+) group, of 11 patients, six took SSRIs, one took only SSRIs, and five took SSRIs with major tranquilizers as augmentation. Three patients took only one major tranquilizer.

These results can be considered consistent because the control analysis showed no differences between the repliers and non-repliers in each group, except for a difference in age of onset between repliers and nonrepliers in the OCD (ASD+) group (Supplementary Tables 1-3).

\section{Symptom Reduction}

The ANOVA of the total Y-BOCS score showed a significant effect for time $(F=110.42, p<0.001)$, indicating a decrease in symptoms from pre-treatment to follow-up. The group effect was significant $(F=5.54, p=0.03)$, with the OCD (ASD+) group exhibiting a higher score than the OCD (ASD-) group. The group $\times$ time interaction was not significant $(F=0.95, p=0.34)$ (Table 5). The 2-way ANOVA showed no significant between-group difference in Y-BOCS score improvement rate. A similar result was found for the Y-BOCS Obsessive and Compulsive scores. There was a significant between-group difference in BDIII scores at follow-up, but the time and group $\times$ time interaction was not significant (Table 5).

\section{Patients' Impressions of Treatment Effect at Follow-Up}

The survey of patients' impressions of treatment showed no remarkable differences between the groups at follow-up. Both groups reported their impressions of how their OC symptoms had changed from the first assessment. In the OCD (ASD-) group, the numbers of patients in each category were as follows: very much better $=7$, much better $=9$, a little better $=3$, and a little worse $=1$. In the OCD (ASD+) group, the results were as follows: much better $=6$, a little better $=4$, and unchanged $=2$. There was no significant between-group difference in those who experienced improvement (5-7) and all others $(0-4)$ (Fisher's exact test, $p=0.5478$ ). Both groups attributed their improvement more to CBT than to pharmacological treatment, and this difference was significant (Table 6).

\section{Discussion}

The aim of this study was to investigate the long-term outcome of CBT among OCD patients with comorbid ASD in a naturalistic psychiatric setting and to investigate their impressions of the treatment effect of CBT.

Both groups showed a significant reduction in total YBOCS scores at follow-up compared with the first assessment, but there was no significant between-group difference in the total Y-BOCS score improvement rate. Nevertheless, the total Y-BOCS score at follow-up in the OCD (ASD+) group was significantly higher than in the OCD (ASD-) group. Further,

Table 5 Changes of symptoms: $\mathrm{OCD}\left(\mathrm{ASD}^{-}\right)$versus (ASD+)

\begin{tabular}{|c|c|c|c|c|c|c|c|c|}
\hline & \multirow{2}{*}{$\begin{array}{l}\text { OCD(ASD-) } \\
N=18 \\
\text { Mean } \pm \text { SD }\end{array}$} & \multirow{2}{*}{$\begin{array}{l}\text { OCD }(\mathrm{ASD}+) \\
N=12 \\
\text { Mean } \pm \text { SD }\end{array}$} & \multicolumn{2}{|c|}{ Group effect } & \multicolumn{2}{|c|}{ Time effect } & \multicolumn{2}{|c|}{ Group $\times$ time effect } \\
\hline & & & $\mathrm{F}$ & $\mathrm{p}$ & $\mathrm{F}$ & $\mathrm{p}$ & $\mathrm{F}$ & $\mathrm{p}$ \\
\hline \multicolumn{9}{|l|}{ Y-BOCS total } \\
\hline $\begin{array}{l}\text { Baseline } \\
\text { Follow-up }\end{array}$ & $\begin{array}{l}30.44 \pm 7.14 \\
12.78 \pm 6.96\end{array}$ & $\begin{array}{l}33.58 \pm 5.55 \\
18.92 \pm 6.69\end{array}$ & 5.54 & 0.03 & 110.42 & $<0.001$ & 0.95 & 0.34 \\
\hline \multicolumn{9}{|c|}{ Y-BOCS Obsessive } \\
\hline $\begin{array}{l}\text { Baseline } \\
\text { Follow-up }\end{array}$ & $\begin{array}{l}15.17 \pm 3.83 \\
6.72 \pm 3.16\end{array}$ & $\begin{array}{l}16.67 \pm 2.90 \\
9.58 \pm 3.32\end{array}$ & 4.61 & 0.04 & 111.65 & $<0.001$ & 0.86 & 0.36 \\
\hline \multicolumn{9}{|c|}{ Y-BOCS Compulsive } \\
\hline $\begin{array}{l}\text { Baseline } \\
\text { Follow-up }\end{array}$ & $\begin{array}{l}15.28 \pm 3.72 \\
6.06 \pm 3.96\end{array}$ & $\begin{array}{l}16.92 \pm 2.81 \\
9.33 \pm 3.55\end{array}$ & 5.96 & 0.02 & 89.24 & $<0.001$ & 0.85 & 0.37 \\
\hline \multicolumn{9}{|l|}{ BDI-II } \\
\hline $\begin{array}{l}\text { Baseline } \\
\text { Follow-up }\end{array}$ & $\begin{array}{l}12.33 \pm 8.40 \\
11.00 \pm 8.33\end{array}$ & $\begin{array}{l}16.25 \pm 12.32 \\
19.25 \pm 11.16\end{array}$ & 5.63 & 0.023 & 0.10 & 0.75 & 0.68 & 0.42 \\
\hline
\end{tabular}

Bold numbers show statistical significance

ASD, Autism Spectrum Disorders; BDI, Beck Depression Inventory; OCD, Obsessive Compulsive Disorder; OCD (ASD-), Obsessive compulsive disorder without co-morbid autism spectrum disorders; (ASD+), Obsessive compulsive disorder with co-morbid autism spectrum disorders; Y-BOCS, Yale-Brown Obsessive-Compulsive Scale 
Table 6 Patients' attribution of their improvement: OCD (ASD-) versus (ASD+)

\begin{tabular}{llll}
\hline & $\begin{array}{l}\text { OCD (ASD-) } N=17 \\
\text { Mean } \pm \text { SD }\end{array}$ & $\begin{array}{l}\text { OCD (ASD+) } N=10 \\
\text { Mean } \pm \text { SD }\end{array}$ & $p$ \\
\hline Pharmacotherapy (Yes/No) & $12 / 5$ & $8 / 2$ & 0.678 \\
Cognitive behavioral therapy & $16 / 1$ & $9 / 1$ & 1.000 \\
(Yes/No) & & & \\
\hline
\end{tabular}

OCD (ASD-), Obsessive compulsive disorder without co-morbid autism spectrum disorders; (ASD+), Obsessive compulsive disorder with co-morbid autism spectrum disorders more patients in the OCD (ASD+) group maintained an OCD diagnosis than in the OCD (ASD-) group.

The OCD (ASD+) group showed a significantly higher rate of symptoms on the Y-BOCS Obsession (hoarding and symmetry) and Compulsion (arranging and hoarding) subscales compared with the OCD (ASD-) group, suggesting that modifications of CBT are needed to treat these symptoms (Walters et al. 2016). This result confirms the findings of previous studies indicating that the frequency of these symptoms is higher in the ASD group (Mataix-Cols et al. 2005; Bejerot 2007). Further, behavioral analysis show that ERP is not applicable for those symptoms because of the absence of clear obsession and related fear. The existence and severity of these symptoms influences CBT outcome, as ERP is a very efficient treatment for OCD. The non-applicability of ERP for some OC symptoms is not immediately obvious from a simple symptom description or categorization (e.g., obsession for symmetry, arranging, and hoarding compulsions). For example, one patient in our OCD (ASD+) group complained that he could not stop washing his hands (he washed them for about $40 \mathrm{~min}$ ) but expressed no clear obsession and no fear; his repeated hand washing was based on the need to repeat the behavior until he felt it was just right. We helped this patient to learn an appropriate, more adaptive way of hand washing through shaping. However, as Table 2 shows, there was no difference between the OCD (ASD+) and the OCD (ASD-) groups in fear of contamination and cleaning compulsion, for which ERP is applicable. Therefore, our results indicate that the OCD (ASD+) group showed two types of OC symptoms, ERP applicable and ERP not applicable (Wikramanayake et al. 2017), with differing rates. Nevertheless, we found a significant reduction in OC symptoms even in the OCD (ASD+) group. Considering the more severe OC symptoms in the OCD (ASD+) group, there is a need to develop a tailored CBT-based treatment for this group.

We also found that the OCD (ASD+) group showed increased depressive symptoms (as measured by the BDI-II) at follow-up, despite the significant reduction in OC symptoms. A probable explanation for the heightened BDI-II scores in the OCD (ASD+) group at follow-up might be because as their OC symptoms improved, these patients experienced greater difficulty in social interaction owing to their autistic symptoms. It is likely that they did not experience these difficulties when their OC symptoms were severe and overwhelmed their daily lives.

There was no significant difference between the OCD (ASD+) and OCD (ASD-) groups in patients' impressions of the treatment effect. Both groups attributed their improvement more to CBT than to pharmacological treatment, and the $\mathrm{OCD}(\mathrm{ASD}+)$ group reported more positive experiences of the CBT treatment.

Although there were no significant between-group differences in most of the demographic and clinical data at followup, surprisingly, the OCD (ASD+) patients reported a higher employment rate (including in sheltered workplaces) than the OCD (ASD-) patients. It is possible that after receiving an ASD diagnosis, the OCD (ASD+) group were given more opportunity to receive governmental support in the form of social support in local communities, such as disability pensions and employment in sheltered workplaces.

At the long-term follow-up, a substantial number of patients were receiving psychiatric treatment, including pharmacological therapy, which might reflect one of the main problems with Japan's mental health system. Because of the shortage of CBT therapists, patients' acceptance of long-term medication, and patients' fear of OC symptom recurrence if they reduce their medication, OCD patients tend to be resigned to long-term psychiatric care.

This study suggests that ERP-applicable OC symptoms in individuals with OCD (ASD+) can be improved by CBT. However, there is a need for a better mental health system that can provide individualized and adapted CBT treatment for patients with OCD (ASD+) and does not rely solely on pharmacological treatment. Considering the association between ASDrelated distress and the severity of OC symptoms in this population, particularly those symptoms for which ERP is not applicable, CBT must be tailored to each patient's ASD trait, such as increased involvement of caregivers, use visual stimuli, selfmonitoring, positive reinforcement, and use of clear language and instructions (Walters et al. 2016; Kose et al. 2018). There also needs to be cooperation with the relevant social networks (e.g., family, schools, workplaces, local community) to help these individuals adapt to the social environment.

The strength of this study was the long follow-up period for the CBT outcome, which is rare in studies using naturalistic treatment settings in Japan. We also included patients' 
impressions of the treatment effect of CBT compared with pharmacological treatment. However, the findings should be interpreted with caution because of the study limitations. The small sample size limits the power and generalizability of the findings. An additional problem is the relatively low rate of response to the follow-up survey. More than half the patients who had joined the comparison study did not reply at followup. This may be because of the high self-stigma related to mental illness among parents and individuals with a psychiatric diagnosis in Japan (Shimotsu and Horikawa 2016). A third limitation is that the Y-BOCS measure used at follow-up comprised a self-report form, as the survey was conducted by mail (at the first assessment, the Y-BOCS was scored by an independent assessor in a semi-structured interview). The use of a self-report Y-BOCS measure may have affected the accuracy of OC symptom severity at follow-up.

\section{Conclusion}

Despite its limitations, this follow-up study has considerable clinical significance. The findings indicate that patients with OCD (ASD+) and OCD (ASD-) who receive CBT in a naturalistic setting show decreased OCD symptoms. The OCD (ASD+) group had higher rates of employment than the OCD (ASD-) group. The results suggest the need for individualized and tailored CBT in naturalistic clinical settings for OCD patients with ASD. Additionally, accurate assessment of the effectiveness of a treatment program requires a consideration of the long-term outcome. Therefore, further studies with larger sample sizes and prospectively arranged long-term follow-up are needed.

Acknowledgments This work was partially supported by the Academic Contrubution from Pfizer Japan Inc. We thank Diane Williams, PhD, from Edanz Group (www.edanzediting.com/ac) for editing a draft of this manuscript.

Author Contributions Study concept and design: Akiko Nakagawa and Eriko Nakatani; acquisition of data: Yoko Hiraoka, Tetsuji Miyazaki, Eriko Nakatani, Mayumi Tomita, Kazuko Yoshioka and Shinji Murakami; statistical analysis and interpretation of data: Hirofumi Nishinaka; drafting of the manuscript: Nora Choque Olsson and Akiko Nakagawa; critical revision of the manuscript: Akiko Nakagawa and Nora Choque Olsson; study supervision: Nora Choque Olsson and Shozo Aoki. All authors read and approved the version to be published.

\section{Compliance with Ethical Standards}

Conflict of Interest The authors declare that they have no conflict of interest.

Ethical Approval All procedures performed in studies involving human participants were in accordance with the ethical standards of the institutional and/or national research committee and with the 1964 Helsinki declaration and its later amendments or comparable ethical standards.
Informed Consent Informed consent was obtained from all study participants.

Open Access This article is distributed under the terms of the Creative Commons Attribution 4.0 International License (http:// creativecommons.org/licenses/by/4.0/), which permits unrestricted use, distribution, and reproduction in any medium, provided you give appropriate credit to the original author(s) and the source, provide a link to the Creative Commons license, and indicate if changes were made.

\section{References}

American Psychiatric Association. (2000). Diagnostic and statistical. Washington, DC: Manual of Mental Disorders-Text Revision.

American Psychiatric Association. (2013). Diagnostic and statistical manual of mental disorders, fifth edition (DSM-5 $\left.{ }^{\mathrm{TM}}\right)$. Washington, DC: American Psychiatric Association.

Arildskov, T. W., Hojgaard, D. R., Skarphedinsson, G., Thomsen, P. H., Ivarsson, T., Weidle, B., et al. (2016). Subclinical autism spectrum symptoms in pediatric obsessive-compulsive disorder. European Child \& Adolescent Psychiatry, 25(7), 711-723. https://doi.org/10. 1007/s00787-015-0782-5.

Baron-Cohen, S., Wheelwright, S., Skinner, R., Martin, J., \& Clubley, E. (2001). The autism-spectrum quotient (AQ): Evidence from Asperger syndrome/high-functioning autism, males and females, scientists and mathematicians. Journal of Autism and Developmental Disorders, 31(1), 5-17.

Beck, A. T., \& Steer, R. A. (1996). Beck depression inventory: Manual. San Antonio: Psychological Corporation.

Bejerot, S. (2007). An autistic dimension: A proposed subtype of obsessive-compulsive disorder. Autism, 11(2), 101-110. https://doi. org/10.1177/1362361307075699.

Bejerot, S., Nylander, L., \& Lindstrom, E. (2001). Autistic traits in obsessive-compulsive disorder. Nordic Journal of Psychiatry, 55(3), 169-176. https://doi.org/10.1080/08039480152036047.

Goodman, W. K., Price, L. H., Rasmussen, S. A., Mazure, C., Fleischmann, R. L., Hill, C. L., Heninger, G. R., \& Charney, D. S. (1989). The Yale-Brown obsessive compulsive scale. I. Development, use, and reliability. Archives of General Psychiatry, 46(11), 1006-1011.

Griffiths, D. L., Farrell, L. J., Waters, A. M., \& White, S. W. (2017). ASD traits among youth with obsessive-compulsive disorder. Child Psychiatry and Human Development, 48(6), 911-921. https://doi. org/10.1007/s10578-017-0714-3.

Kamio, Y., Yukihiro, T., Adachi, J., Ichikawa, H., Inoue, M., Uchiyama, T., et al. (2006). Checklist for behavior on pervasive developmental disorder among adolescents and adults: Reliability and validity of pervasive developmental disorders autism society Japan rating scale (PARS). Seishin Igaku, 48, 495-505. (in Japanese).

Kohn, R., Saxena, S., Levav, I., \& Saraceno, B. (2004). The treatment gap in mental health care. Bull World Health Organ, 82(11), 858-866.

Kojima, M., Furukawa, T. A., Takahashi, H., Kawai, M., Nagaya, T., \& Tokudome, S. (2002). Cross-cultural validation of the Beck depression inventory-II in Japan. Psychiatry Research, 110(3), 291-299.

Kose, L. K., Fox, L., \& Storch, E. A. (2018). Effectiveness of cognitive behavioral therapy for individuals with autism spectrum disorders and comorbid obsessive-compulsive disorder: A review of the research. Journal of Developmental and Physical Disabilities, 30(1), 69-87. https://doi.org/10.1007/s10882-017-9559-8.

Maddox, B. B., Miyazaki, Y., \& White, S. W. (2016). Long-term effects of CBT on social impairment in adolescents with ASD. Journal of 
Autism and Developmental Disorders., 47, 3872-3882. https://doi. org/10.1007/s10803-016-2779-4.

Mataix-Cols, D., Rosario-Campos, M. C., \& Leckman, J. F. (2005). A multidimensional model of obsessive-compulsive disorder. The American Journal of Psychiatry, 162(2), 228-238. https://doi.org/ 10.1176/appi.ajp.162.2.228.

Meier, S. M., Petersen, L., Schendel, D. E., Mattheisen, M., Mortensen, P. B., \& Mors, O. (2015). Obsessive-compulsive disorder and autism spectrum disorders: Longitudinal and offspring risk. PLoS One, 10(11), e0141703. https://doi.org/10.1371/journal.pone.0141703.

Mito, H., Matsuura, N., Mukai, K., Yanagisawa, Y., Nakajima, A., Motoyama, M., Arikawa, A., Yamanishi, K., \& Matsunaga, H. (2014). The impacts of elevated autism spectrum disorder traits on clinical and psychosocial features and long-term treatment outcome in adult patients with obsessive-compulsive disorder. Comprehensive Psychiatry, 55(7), 1526-1533. https://doi.org/10. 1016/j.comppsych.2014.05.005.

Murray, K., Jassi, A., Mataix-Cols, D., Barrow, F., \& Krebs, G. (2015). Outcomes of cognitive behaviour therapy for obsessive-compulsive disorder in young people with and without autism spectrum disorders: A case controlled study. Psychiatry Research, 228(1), 8-13. https://doi.org/10.1016/j.psychres.2015.03.012.

O'Neill, J., \& Feusner, J. D. (2015). Cognitive-behavioral therapy for obsessive-compulsive disorder: Access to treatment, prediction of long-term outcome with neuroimaging. Psychology Research and Behavior Management, 8, 211-223. https://doi.org/10.2147/prbm. s75106.

Otsubo, T., Tanaka, K., Koda, R., Shinoda, J., Sano, N., Tanaka, S., et al. (2005). Reliability and validity of Japanese version of the miniinternational neuropsychiatric interview. Psychiatry and Clinical Neurosciences, 59(5), 517-526. https://doi.org/10.1111/j.14401819.2005.01408.x.

Piacentini, J., Bergman, R. L., Keller, M., \& McCracken, J. (2003). Functional impairment in children and adolescents with obsessivecompulsive disorder. Journal of Child and Adolescent Psychopharmacology, 13(Suppl 1), S61-S69. https://doi.org/10. 1089/104454603322126359.

Russell, A. J., Jassi, A., Fullana, M. A., Mack, H., Johnston, K., Heyman, I., Murphy, D. G., \& Mataix-Cols, D. (2013). Cognitive behavior therapy for comorbid obsessive-compulsive disorder in highfunctioning autism spectrum disorders: A randomized controlled trial. Depression and Anxiety, 30(8), 697-708. https://doi.org/10. 1002/da.22053.

Ruta, L., Mugno, D., D'Arrigo, V. G., Vitiello, B., \& Mazzone, L. (2010). Obsessive-compulsive traits in children and adolescents with Asperger syndrome. European Child \& Adolescent Psychiatry, 19(1), 17-24. https://doi.org/10.1007/s00787-009-0035-6.

Sharma, E., Thennarasu, K., \& Reddy, Y. C. (2014). Long-term outcome of obsessive-compulsive disorder in adults: A meta-analysis. The Journal of Clinical Psychiatry, 75(9), 1019-1027. https://doi.org/ 10.4088/JCP.13r08849.

Shimotsu, S., \& Horikawa, N. (2016). Self-stigma in depressive patients: Association of cognitive schemata, depression, and self-esteem. Asian Journal of Psychiatry, 24, 125-129. https://doi.org/10.1016/ j.ajp.2016.09.003.

Wakabayashi, A., Baron-Cohen, S., Wheelwright, S., \& Tojo, Y. (2006). The autism-spectrum quotient (AQ) in Japan: A cross-cultural comparison. Journal of Autism and Developmental Disorders, 36(2), 263-270. https://doi.org/10.1007/s10803-005-0061-2.

Walters, S., Loades, M., \& Russell, A. (2016). A systematic review of effective modifications to cognitive Behavioural therapy for young people with autism Spectrum disorders. Review Journal of Autism and Developmental Disorders, 3(2), 137-153. https://doi.org/10. 1007/s40489-016-0072-2.

Wechsler, D. (1997). The Wechsler adult intelligence scale- third edition (WAIS-III) administration and scoring manual. San Antonio: The Psychological Corporation.

White, S. W., Ollendick, T., Albano, A. M., Oswald, D., Johnson, C., Southam-Gerow, M. A., Kim, I., \& Scahill, L. (2013). Randomized controlled trial: Multimodal anxiety and social skill intervention for adolescents with autism spectrum disorder. Journal of Autism and Developmental Disorders, 43(2), 382-394. https://doi.org/10.1007/ s10803-012-1577-x.

Wikramanayake, M., Mandy, W., Shahper, S., Kaur, S., Kolli, S., Osman, S., et al. (2017). Autism Spectrum disorders in adult outpatients with obsessive compulsive disorder in the UK. International Journal of Psychiatry in Clinical Practice, 22(1), 54-62. https://doi.org/10. 1080/13651501.2017.1354029.

Yamashita Y. (2010). Clinical features of obsessive-compulsive disorder with pervasive developmental disorder. Seishin Shinkeigaku Zasshi, $112,853-866$. 